\title{
Problematic Alcohol and Drug Use Is Associated with Low Self-Directedness and Cooperativeness
}

\author{
Steinn Steingrimsson ${ }^{a, b}$ Hanne Krage Carlsen ${ }^{b}$ Emil Lundström ${ }^{c}$ \\ Sebastian Lundström ${ }^{\mathrm{a}}$ Thomas Nilsson ${ }^{\mathrm{a}, \mathrm{d}}$ \\ ${ }^{a}$ Centre of Ethics, Law and Mental Health (CELAM), University of Gothenburg, Gothenburg, Sweden; b Sahlgrenska \\ Academy, University of Gothenburg, Gothenburg, Sweden; ' Adult Psychiatric Clinic, Södra Älvsborg Hospital Borås, \\ Borås, Sweden; d Department of Forensic Psychiatry, National Board of Forensic Medicine, Gothenburg, Sweden
}

\section{Keywords}

Alcohol use · Drug use · Epidemiology · Adolescents ·

Personality traits

\begin{abstract}
Background: Personality traits, such as self-directedness (SD) and cooperativeness (CO), may be indicative of problematic alcohol and/or drug use. Objectives: The aim of this study was to quantify the association of substance use with SD and CO in a large cohort of adolescents. Method: A total of 6,917 individuals (58\% women) at the age of 18 who had filled in the Alcohol Use Disorder Identification Test (AUDIT) and Drug Use Disorder Identification Test (DUDIT), and the SD and CO scales from the Temperament and Character Inventory, as part of the Child and Adolescent Twin study in Sweden were included in the analyses. Results: High AUDIT scores $(>15)$ were found in $2.4 \%$ of the population and high DUDIT scores $(>7)$ in $1.2 \%$ of the population. Total score on the AUDIT was negatively correlated ( $p<0.001)$ with SD $(r=-0.18)$ and CO $(r=-0.15)$, as well as total DUDIT with SD $(r=-0.11)$ and CO $(r=-0.08)$. The risk of high AUDIT $(>15)$ and DUDIT ( $>7$ ) was highest for those with a low (1 standard deviation below mean) SD score (ORs 4.1 and 4.5, $p<0.001$ ) and a low CO score (ORs 3.5 and 4.5, $p<0.001$ ). However, at
\end{abstract}

karger@karger.com www.karger.com/ear

Karger $\stackrel{\text { ' }}{5}$

GOPEN ACCESS
(C) 2020 The Author(s)

Published by S. Karger AG, Basel

This is an Open Access article licensed under the Creative Commons Attribution-NonCommercial-4.0 International License (CC BY-NC) (http://www.karger.com/Services/OpenAccessLicense), applicable to the online version of the article only. Usage and distribution for commercial purposes requires written permission.
1 standard deviation above mean, no association between alcohol or drug use and SD or CO was seen. Using SD and CO scores to predict AUDIT $>15$ or DUDIT $>7$ yielded a sensitivity between 62.4 and $71.3 \%$ and a specificity between 64.9 and $70.4 \%$. Conclusions: Personality traits of low SD and CO are associated with increased alcohol and drug use. These findings support the notion that personality traits can be used to identify individuals at high risk of substance abuse.

(c) 2020 The Author(s)

Published by S. Karger AG, Basel

\section{Introduction}

Alcohol and drug use are among the leading factors behind increased mortality and morbidity worldwide [1]. The pathogenesis of substance use disorders (SUDs) is multifactorial, being influenced by both genetic and environmental factors $[2,3]$. Furthermore, deviant personality traits are common among individuals with SUDs, manifested, for example, by a high rate of personality disorders in this group [4].

Personality traits are distributed on a continuum, varying between individuals and can fluctuate over time [5]. Although genetics explain a large part of the variation 
in personality traits, both non-shared and shared environmental factors are substantially implicated. The Temperament and Character Inventory (TCI) subscales selfdirectedness (SD) and cooperativeness (CO) reflect individual differences in goals and values that influence choices and intentions as well as the overall sense of direction of one's life, and together they represent what could be labeled as an individual's character maturity [6]. The subscales of SD and $\mathrm{CO}$ have been shown to be a marker for personality disorders [7] and have also been found to be lower among those that abuse drugs [8]. Treatment outcome is also related to differences in $\mathrm{SD}$ and $\mathrm{CO}$ scores, where, for example, higher scores predicted a better treatment prognosis among patients with alcohol use disorder, especially outpatient treatment adherence after detoxification [9].

However, the association of problematic alcohol and drug use with SD and CO is generally based on individuals sampled from clinical settings. Thus, both from a predictive and preventive perspective, population-based studies are warranted. Indeed, intervention studies have shown that low SD and CO are malleable to treatment, using, for example, meditation based on mindfulness [10] and psychotherapeutic efforts [11]. This provides a need for further quantifying the association between use of alcohol and drugs and scores on SD and CO at the population level. An individual's SD and CO scores could be an important factor, by directly or indirectly, influencing their risk of initiating a trajectory leading up to abuse, and changing this score through treatment interventions could be important for clinical management of ongoing abuse.

The aim of this study was to quantify the association between SD and CO with self-reported alcohol and drug use in a large prospective population-based cohort of Swedish 18 -year-old twins.

\section{Materials and Methods}

\section{Subjects}

The participants were recruited from the ongoing Child and Adolescent Twin study in Sweden (CATSS). CATSS is described in detail elsewhere [12]. Put briefly, parents to all twins born in Sweden from July 1, 1992, and onward are invited to participate in the study (telephone interview) at the 9th birthday. At age 18, twins and their parents were contacted again to participate in the CATSS-18 by logging on to a website and filling out a web questionnaire containing several instruments, among these the Alcohol Use Disorder Identification Test (AUDIT), Drug Use Disorder Identification Test (DUDIT), and the 2 scales of SD and CO from the TCI. The response rate in CATSS-18 has been around $50 \%$ up to this date. Two previous papers from the same cohort have looked at AUDIT and DUDIT; however, none of the articles includes the TCI as exposure or even included it in the analyses [13, $14]$.

\section{Measures and Procedure}

Alcohol use was assessed with AUDIT, a screening instrument containing 10 questions concerning alcohol habits developed by the World Health Organization [15]. The scale contains questions on frequency, quantity, control of drinking, and social consequences. All questions are rated from 0 to 4 points indicating the least to most severe abuse. Adjusted AUDIT scores were divided into 3 categories: "low risk" (0-7 points), "risky" (8-15 points), and "high risk or likely dependent" ( $>15$ points), according to the World Health Organization recommendations [15].

Non-prescribed drug use was assessed using DUDIT, a screening instrument with a total of 11 questions with the same scoring as AUDIT. This scale has favorable reliability, sensitivity, and specificity; however, cutoff points are not well defined [16], and since the use of drugs was less common than the use of alcohol, lower scores were used to define the categories. DUDIT scores were divided into "no use" (DUDIT =0), "use" (DUDIT between 1 and 7 ), and "risky use" (DUDIT >7).

TCI subscales of SD and CO were used to measure personality traits related to character maturity [6]. SD reflects to what degree an individual conceptualizes himself/herself as an autonomous person in control of their own destiny [6]. CO mirrors to what extent an individual conceptualizes himself/herself in terms of an integral part of the human society, comprising feelings of compassion, conscience, and a will to interact with others. Both scales, consisting of 25 items each, are coded in a binary fashion with " 1 " or " 0 " corresponding to answers of "true" or "false", where " 1 " gives a one-point increase on the scale. The SD and CO values were standardized into regular $t$-scores with a mean of 50 and a standard deviation of \pm 10 . These 2 subscales were the only ones from TCI that were included in the data collection and therefore reported.

\section{Procedures for Missing Values}

In order to include only individuals with valid answers, the following procedures were performed for each of the variables:

- AUDIT: The first question was a gate question that asks about any alcohol use. If the response is "no alcohol use," then no further questions are given, that is, if an individual answered 0 (meaning no alcohol consumption), the total score was 0 . If an individual had $>2$ unanswered questions, the individual was excluded from the analysis. However, if an individual answered 8 , 9 , or 10 answers, an adjusted score was calculated by adding the sum of the answers and multiplying by 10 divided by the number of given answers. A total of 7,220 had a valid AUDIT score.

- DUDIT: If an individual answered "0" (meaning no drug consumption), the total score was 0 . If an individual with a score of " 1 " on the first questions had $>2$ unanswered questions, he or she was excluded from further analysis on DUDIT. If an individual answered 9, 10, or 11 questions, an adjusted score was calculated by adding the sum of the answers and multiplying by 11 divided by the number of questions answered. A total of 7,249 had a valid score.

- SD and CO: Each scale score was calculated as the sum of questions that an individual answered with a "true" statement and included if no $>2$ questions were unanswered. Valid scores were available for 7,311 on SD and 7,097 for CO. 
Table 1. AUDIT, DUDIT, SD, and CO among twins at 18 years

\begin{tabular}{|c|c|c|c|c|}
\hline & Total $(n=6,917)$ & Male $(n=2,901)$ & Female $(n=4,016)$ & $p$ value* \\
\hline AUDIT & $4.8 \pm 4.3 ; 4.0(0-34)$ & $5.1 \pm 4.6 ; 5.0(0-34)$ & $4.5 \pm 4.1 ; 4.0(0-28)$ & $<0.001$ \\
\hline $0-7$ & $5,500(79.5)$ & $2,210(76.2)$ & $3,290(81.9)$ & $<0.001$ \\
\hline $8-15$ & $1,253(18.1)$ & $612(21.1)$ & $641(16.0)$ & \\
\hline$>15$ & $164(2.4)$ & $79(2.7)$ & $85(2.1)$ & \\
\hline DUDIT & $0.3 \pm 1.6 ; 0.0(0-30)$ & $0.4 \pm 1.8 ; 0.0(0-29)$ & $0.2 \pm 1.5 ; 0.0(0-30)$ & $<0.001$ \\
\hline 0 & $6,555(94.8)$ & $2,694(92.9)$ & $3,861(96.1)$ & $<0.001$ \\
\hline $1-7$ & $277(4.0)$ & $157(5.4)$ & $120(3.0)$ & \\
\hline$>7$ & $85(1.2)$ & $50(1.7)$ & $35(0.9)$ & \\
\hline $\mathrm{SD}$ & $15.4 \pm 3.6 ; 16.0(0-25)$ & $15.8 \pm 3.5 ; 16.0(0-25)$ & $15.1 \pm 3.6 ; 16.0(2-25)$ & $<0.001$ \\
\hline T normalized & $50.0 \pm 9.9 ; 51.5(7.4-76.4)$ & $51.1 \pm 9.7 ; 51.5(7.4-76.4)$ & $49.2 \pm 10.0 ; 51.6(12.9-76.4)$ & $<0.001$ \\
\hline $\mathrm{CO}$ & $13.9 \pm 3.3 ; 14.0(0-25)$ & $14.1 \pm 3.5 ; 14.0(0-25)$ & $13.8 \pm 3.2 ; 14.0(1-25)$ & $<0.001$ \\
\hline T normalized & $50.0 \pm 9.8 ; 50.1(8.9-82.4)$ & $50.5 \pm 10.2 ; 50.0(8.9-82.4)$ & $49.6 \pm 9.5 ; 50.1(11.9-82.4)$ & $<0.001$ \\
\hline
\end{tabular}

Continuous variables presented as mean $\pm \mathrm{SD}$; median (range) and categorical variables as $n(\%)$.

* $t$ test of mean and chi-square test of equal distributions for categorical values.

SD, self-directedness; CO, cooperativeness; AUDIT, Alcohol Use Disorder Identification Test; DUDIT, Drug Use Disorder Identification Test.

In summary, individuals who responded to at least 8 and 9 questions on the AUDIT and DUDIT scales, respectively, and at least 23 of 25 questions in the SD and CO scales were included. A total of 180 of 7,619 individuals (2.4\%) had no valid score on any of the 4 variables and were not included in the study population. Of the remaining 7,439 individuals, 6,917 (93.0\%), of which 2,901 (41.9\%) were male and 4,016 (58.1\%) were female, had valid scores on all 4 variables. For each cross-correlation, the number of individuals on both variables needed to have valid scores and the number of analyzed subjects is given in each case.

Analysis

First summary statistics were calculated for the outcome variables for individuals with valid scores. Score differences between males and females were calculated using $t$ test for continuous variables and $\chi^{2}$ tests for categorical values.

Then for analysis of linear association, a pairwise Pearson's correlation coefficient was calculated between the total scores on (a) AUDIT, (b) DUDIT, (c) SD and (d) CO and presented with 95\% CIs. In order to investigate nonlinear associations between the variables, spline models were drawn and analyzed with the distributed lag non-linear models package in R [17] for each pair of outcome variables. Degrees of freedom for the modeling of splines were chosen based on minimizing the residuals and were 4 in every case except for the correlation between CO and DUDIT where 7 degrees of freedom gave the best fit. The predicted outcomes from these models were plotted across the $t$-scores of SD and CO, respectively, with the spline centered at 50 (the mean value).

Furthermore, logistic regression was used to calculate the OR of belonging to the highest risk groups of AUDIT $(>15)$ and DUDIT $(>7)$, with independent variables of 1 standard deviation higher and lower of SD and $\mathrm{CO}$ where the middle category was used as the reference category. The ORs were reported with the 95\% CI. The categorical association was further illustrated with box-plots with the mean of SD and CO plotted against level of use according to AUDIT or DUDIT.
Lastly, receiver operating curves (ROC) were used to identify the predictive value of SD and CO score (and their composite score) as independent variables for the dependent variables AUDIT $>15$ and DUDIT $>7$. The area under the curve (AUC) is a measure of the overall predictive validity of the independent variable where 0.5 equals random prediction and 1 a perfect prediction. From the ROC analyses, we determined the optimal value of $\mathrm{SD}$ and $\mathrm{CO}$ for predicting scores of AUDIT $>15$ and DUDIT $>7$. The optimal value was defined as the value with both specificity and sensitivity closest to 1 .

All statistical analyses were performed using R 3.4 [18]. A $p$ value $<0.05$ was considered statistically significant in all calculations or if a confidence interval did not intersect with the reference value, respectively.

\section{Results}

\section{Participants}

A total of 6,917 individuals completed all questionnaires and were included in the study. The most common self-reported use of alcohol was $0-7$ points on AUDIT ("low risk"), and the self-reported use of drugs was most often 0 points on DUDIT ("no use", Table 1). There was a statistically significant sex difference, with males scoring higher on both AUDIT and DUDIT compared to females $(p<0.001)$. This difference was reflected in the high-risk categories with AUDIT risk of "high risk/likely dependent" being 2.7\% among males and 2.1\% among females $(p<0.001)$, and for DUDIT "risky use," the rate was 1.7 and $0.9 \%$ for males and females, respectively $(p<$ $0.001)$. 
Table 2. Pearson's correlation coefficients with confidence intervals between scores of AUDIT, DUDIT, SD, and CO among adolescents scores at 18 years, $p<0.0001$ in all correlations

\begin{tabular}{lccc}
\hline & AUDIT & DUDIT & SD \\
\hline AUDIT & 1 & & \\
DUDIT & $0.29(0.26$ to 0.31$)$ & 1 & 1 \\
SD (tci1) & $-0.20(-0.22$ to -0.17$)$ & $-0.11(-0.14$ to -0.09$)$ & $0.63(0.61$ to -0.064$)$ \\
CO (tci2) & $-0.16(-0.18$ to -0.14$)$ & $-0.09(-0.11$ to -0.06$)$ & 1 \\
\hline
\end{tabular}

AUDIT, Alcohol Use Disorder Identification Test Score; DUDIT, Drug Use Disorder Identification Test Score; SD, self-directedness; $\mathrm{CO}$, cooperativeness.

Fig. 1. Nonlinear association between personality traits and scores of AUDIT and DUDIT drawn with a distributed lag nonlinear model. AUDIT, Alcohol Use Disorder Identification Test; DUDIT, Drug Use Disorder Identification Test; CO, cooperativeness.

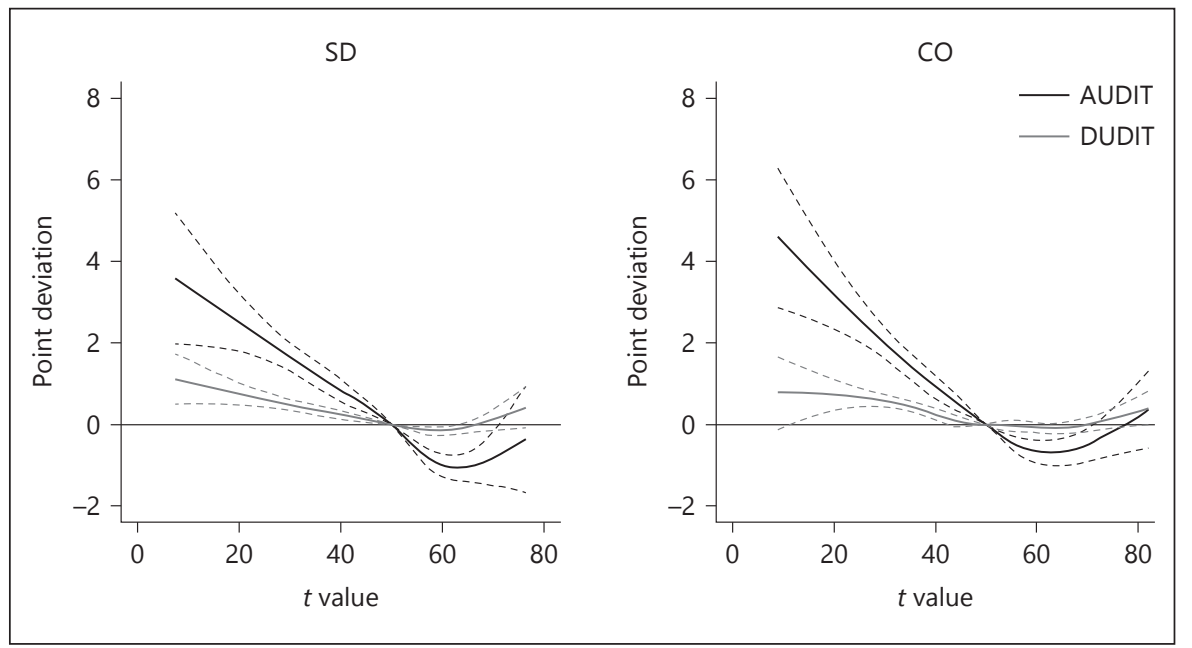

with similar results. However, the number of individuals that scored 2 standard deviations above the mean is small due to skewedness in the data. Furthermore, the cumulated effect of under 1 standard deviation below the mean of both SD and CO scores accentuated the risk for risky use, whereas higher scores did not have a cumulative effect (online suppl. Table 1; for all online suppl. material, see www.karger.com/doi/10.1159/000506473).

To control for twin bias, a separate analysis of Table 3 was done using only a single member in a twin pair as shown in online supplementary Table 2 showing that the main results remained similar albeit numerically different.

\section{Sensitivity and Specificity Analysis}

The AUC for both AUDIT and DUDIT were best for the composite score of SD and CO with AUC 72.9\% for AUDIT and $71.9 \%$ for DUDIT (Fig. 3). For AUDIT, the optimal predictive scores were $\mathrm{SD}=12$ (sensitivity $67.7 \%$, specificity $65.3 \%$ ), $\mathrm{CO}=14$ (sensitivity $63.4 \%$, specificity $69.4 \%$ ), and composite score $=24$ (sensitivity 
Fig. 2. Association between drug and alcohol use risk groups and SD and CO scores. $\mathrm{CO}$, cooperativeness; SD, self-directedness; AUDIT, Alcohol Use Disorder Identification Test; DUDIT, Drug Use Disorder Identification Test.

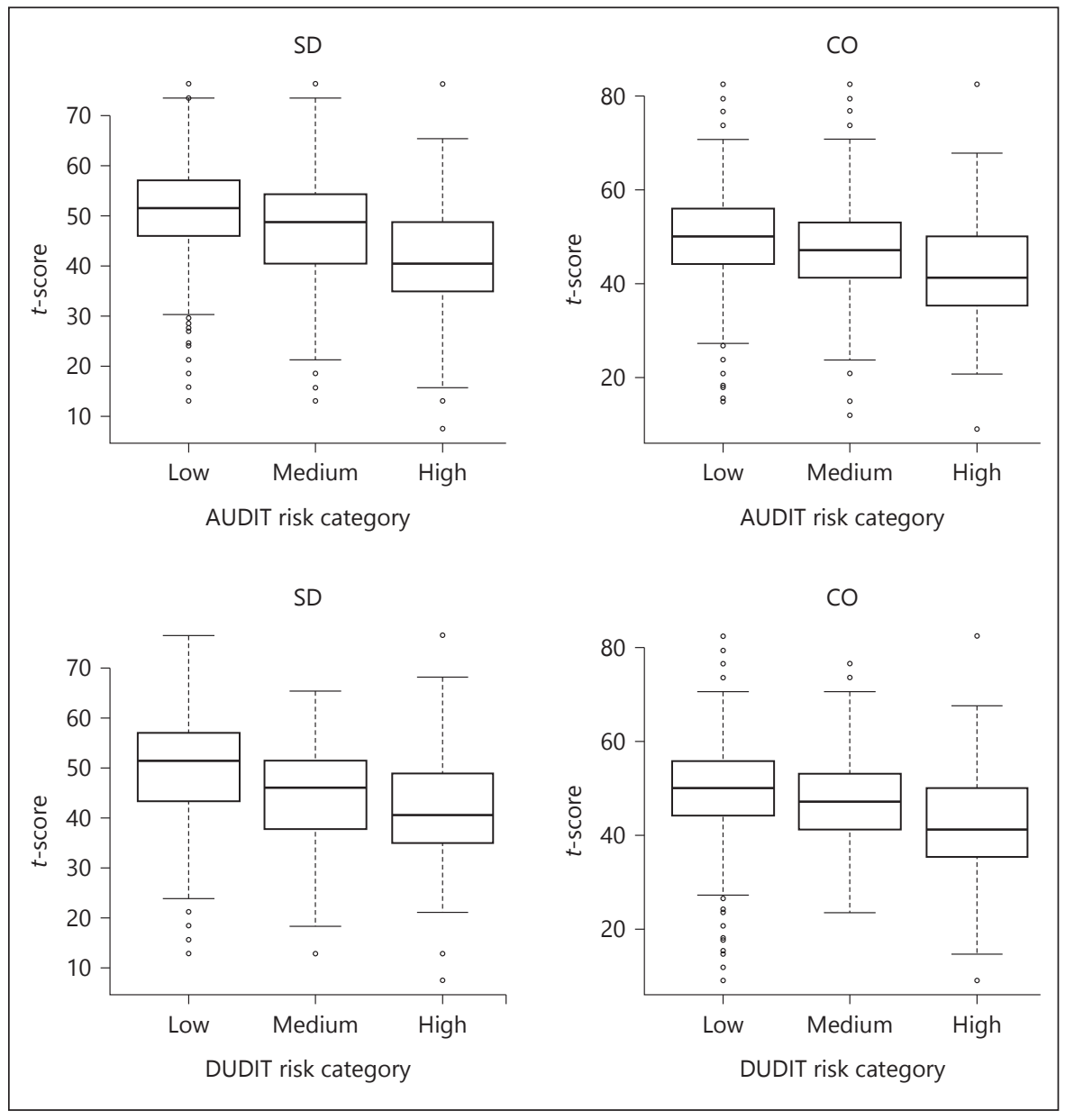

Table 3. Risk of high alcohol use (AUDIT >15) by categories of SD and CO (row-wise percentages)

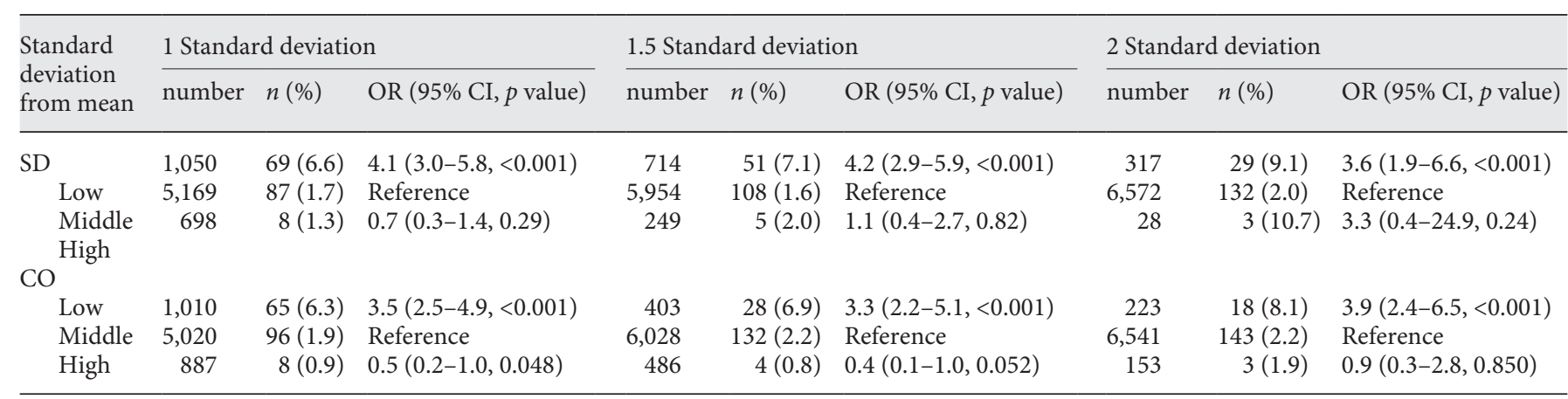

AUDIT, Alcohol Use Disorder Identification Test Score; CO, cooperativeness; SD, self-directedness.

$71.3 \%$, specificity $65.9 \%$ ). For DUDIT, the optimal predictive scores were $\mathrm{SD}=12$ (sensitivity $69.4 \%$, specificity $64.9 \%$ ), $\mathrm{CO}=14$ (sensitivity $62.4 \%$, specificity $69.0 \%$ ), and composite score $=25$ (sensitivity $65.9 \%$, specificity $70.4 \%)$.

\section{Discussion/Conclusion}

The character traits SD and CO were robustly associated with AUDIT and DUDIT scores in the present study, in particular, low scores on SD and $\mathrm{CO}$ with higher use of 


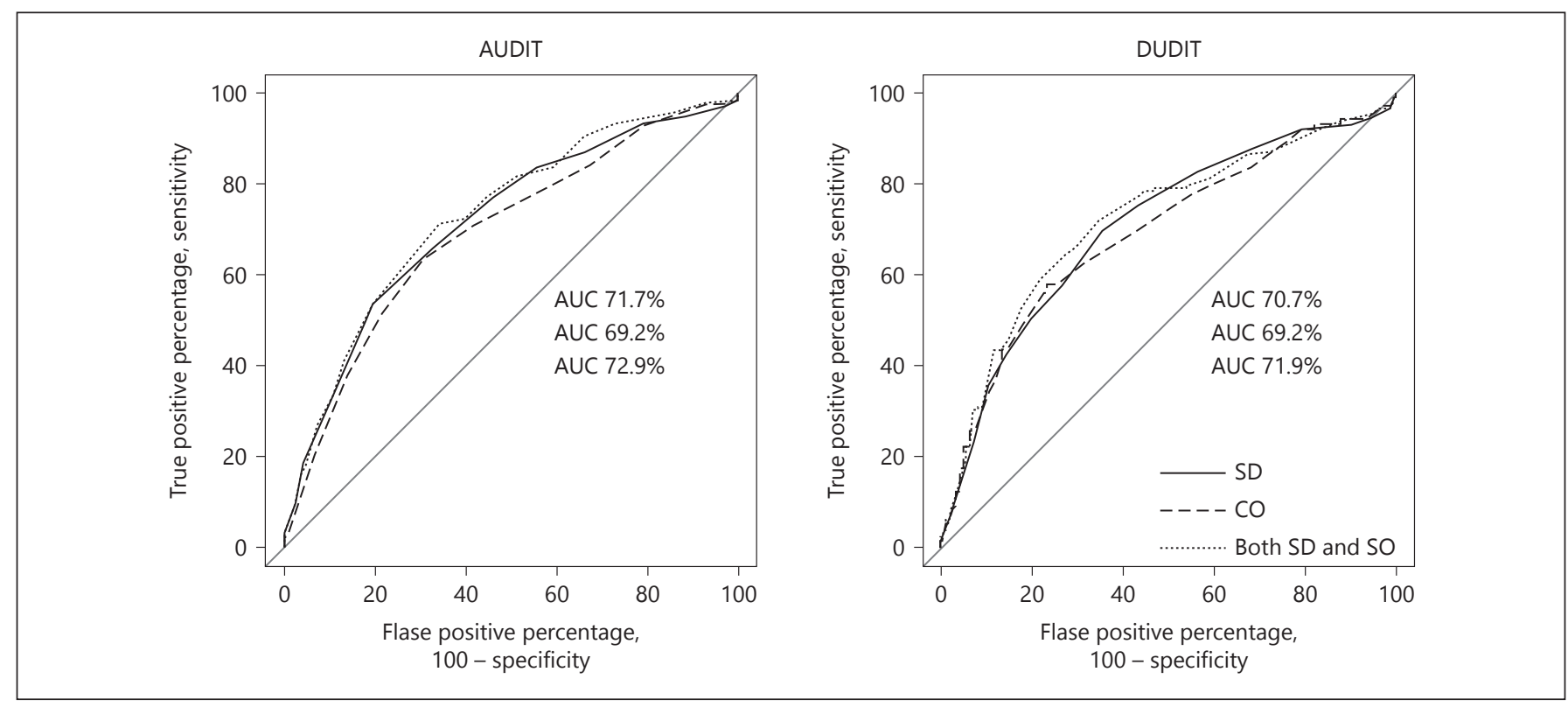

Fig. 3. ROCs for prediction of AUDIT $>15$ and DUDIT $>7$ based on SD and CO scores. AUDIT, Alcohol Use Disorder Identification Test; DUDIT, Drug Use Disorder Identification Test; AUC, area under the curve; CO, cooperativeness; SD, self-directedness.

Table 4. Risk of high drug use (DUDIT >7) by categories of SD and CO (row-wise percentages)

\begin{tabular}{|c|c|c|c|c|c|c|c|c|c|}
\hline \multirow{2}{*}{$\begin{array}{l}\text { Standard } \\
\text { deviation } \\
\text { from mean }\end{array}$} & \multicolumn{3}{|c|}{1 Standard deviation } & \multicolumn{3}{|c|}{ 1.5 Standard deviation } & \multicolumn{3}{|c|}{2 Standard deviation } \\
\hline & number & $n(\%)$ & OR (95\% CI, $p$ value) & number & $n(\%)$ & OR (95\% CI, $p$ value) & number & $n(\%)$ & OR (95\% CI, $p$ value) \\
\hline \multicolumn{10}{|l|}{$\mathrm{SD}$} \\
\hline Low & 1,050 & $37(3.5)$ & $4.5(2.9-7.0,<0.001)$ & 714 & $51(7.1)$ & $5.1(3.2-8.0,<0.001)$ & 317 & $29(9.1)$ & $3.6(1.9-6.6,<0.001)$ \\
\hline Middle & 5,169 & $42(0.8)$ & Reference & 5,954 & $108(1.6)$ & Reference & 6,572 & $132(2.0)$ & Reference \\
\hline High & 698 & $6(0.9)$ & $1.1(0.4-2.5,0.90)$ & 249 & $5(2.0)$ & $1.9(0.7-5.3,0.22)$ & 28 & $3(10.7)$ & $3.3(0.4-24.9,0.24)$ \\
\hline \multicolumn{10}{|l|}{$\mathrm{CO}$} \\
\hline Low & 1,010 & $37(3.7)$ & $4.5(2.9-7.0,<0.001)$ & 403 & $28(6.9)$ & $4.5(2.6-7.7,<0.001)$ & 223 & $8(3.6)$ & $3.2(1.5-6.7,0.002)$ \\
\hline Middle & 5,020 & $42(0.8)$ & Reference & 6,028 & $132(2.2)$ & Reference & 6,541 & $75(1.1)$ & Reference \\
\hline High & 887 & $6(0.7)$ & $0.8(0.3-1.9,0.63)$ & 486 & $4(0.8)$ & $1.0(0.4-2.5,1.00)$ & 153 & $2(1.3)$ & $1.1(0.3-4.7,0.85)$ \\
\hline
\end{tabular}

DUDIT, Drug Use Disorder Identification Test Score; CO, cooperativeness; SD, self-directedness.

alcohol and drugs. Previous studies generally associate lower SD and CO scores to problematic substance use consistent with our findings, although $\mathrm{CO}$ has been suggested to vary with the substance in question [19]. Furthermore, an interesting finding in the present study is that higher scores on SD and CO were not associated with lower alcohol and drug use, suggesting that there may be a threshold where increased scores on character traits have no protective effect.

Results from cross-sectional cohort studies, such as the present study, cannot be used to infer direct causality between the examined variables but depict patterns of associations between them. In accordance with these patterns, the relationship between character and substance use emerges as bidirectional. The consumption of substances is likely to affect the individuals' general reaction to the environment and may therefore cause a change in the character traits or the measurement of them. Also, other psychopathology may interact; a recent study found that among treatment-seeking patients with alcohol use disorder, higher SD and CO scores were associated with a decreased risk of relapse; however, when severity of depression was included in the analyses, the association was minimal [20]. which might suggest that lower scores on SD and $\mathrm{CO}$ indicate depressive symptoms. Indeed, low scores of SD and CO can be seen as a 
general marker of mental health problems [7]. Furthermore, the correlations in the present study were weak with scores ranging between -0.09 and -0.2 , meaning that these variables explain around $10-20 \%$ of the variation in the group. However, the risk of high alcohol and/ or drug use was strongly predicted by low scores on SD and $\mathrm{CO}$ (Tables 3,4$)$ as well as attenuated if both are low in combination (online suppl. Table 1). Another possible explanation might be early-onset neurodevelopmental disorders such as autism and attention-deficit/hyperactivity disorder. These disorders have previously been shown to correlate with lower SD and CO scores [21], which could indicate that at 18 years of age, the use of alcohol and drugs is higher among those already experiencing problems with social interaction and executive functioning. Other markers have been shown to correlate with SD/CO traits such as Machiavellianism, which was shown to differ in groups of controls and cocaine users similar to our study [22].

The sex differences were significant for both AUDIT and DUDIT in the present study, but for AUDIT, the rates of "high risk or likely dependent" were numerically similar between males and females by 2.7 and $2.1 \%$, respectively. For DUDIT, the rate of "risky use" was proportionally larger in males; nevertheless, high-risk scores were rare in both males and females: 1.7 and $0.9 \%$, respectively. In recent years, there has been a tendency of a convergence in the rate of problematic alcohol use between the sexes in many countries [23, 24]. However, this is not consistently so for drugs, where, for example, the gender gap of cannabis use may have widened in the US [25].

The results of this study suggest SD and CO as possible intervention targets for both primary prevention and treatment settings. This is in line with previous research where lower SD and CO scores predicted higher dropout rates for follow-up during outpatient SUD treatment in the first 100 days after inpatient detoxification [9]. In other addictive disorders, low SD has been shown to be predictive of, for example, problematic internet use [26] as well as high alcohol use among treatment-seeking gambling disorder patients [27]. However, in substance abuse, the relationship between SD and $\mathrm{CO}$ and abuse severity can vary as shown in a study by Zaaijer et al. [28], where dependent opiate users had lower SD scores than nondependent opiate users. Both groups of opiate users had similar CO scores, but both $\mathrm{SD}$ and $\mathrm{CO}$ scores were lower than in controls. Recently, it has been shown that SD and CO in healthy individuals are malleable to treatment, for example, mind- fulness [10]. Interventions aimed at increasing SD and CO scores may thus provide a complementary approach to general addiction treatment. Furthermore, there is a debate whether lower cutoff point on scales for alcohol use should be applied to adolescents, for example, when AUDIT was compared to a structured interview, a score of 5 correlated optimally with problematic alcohol use [29]. Clinical samples could provide a clearer picture of which cutoff points to use on each scale to identify personal co-variation and thus give sensitivity and specificity. Our results might, however, suggest that SD and CO are more applicable to a group level than on an individual clinical level since the ROC-analysis found the best predictive value of the composite scores to be $72.9 \%$ for AUDIT and 71.9\% for DUDIT, which can be considered as fair. An issue is that the score on the SD and $\mathrm{CO}$ scales was rather high and therefore not very useable in a clinical setting.

Generalizability of the findings might be influenced by the fact that only twins were included as study subjects since twins may have higher character trait scores than non-twin study subjects [5]. However, an eventually enhanced level of total score would not discard the robust linear and categorical associations found in this study, and the stepwise increase in Figures 1 and 2 suggests that the relationship is linear for lower SD and CO scores, making eventual bias from different absolute score values irrelevant. Furthermore, in online supplementary Table 2 , an analysis of only single twins from a pair shows the relationship of low SD and $\mathrm{CO}$ to be associated with risky alcohol and drug use, while higher scores are not statistically associated. A minor limitation is that out of everyone invited to participate only $60 \%$ were included in the analyses, and this reduction might have led to some bias affecting the results.

Another limitation is that the DUDIT scale is not specific to the type of substances, meaning that answers can pertain to anything from nonmedical use of prescription medication like methylphenidate or smoking of cannabis to intravenous use of opiates. However, the total use of any drugs without prescription indicates problematic drug use, although health, social, and other consequences of use of different substances vary greatly, not only between drugs but also between different social and cultural settings. Finally, a limitation of using 2 subscales of TCI should be mentioned since other subscales could be of interest; however, these were not applied in the data collection and could be included in future studies of the relationship between substance use and TCI. 


\section{Conclusion}

In this large cohort study, lower scores of SD and CO were weakly but significantly associated with higher risk of problematic alcohol and drug use, whereas higher scores were not necessarily protective. These findings could be used to guide individual treatment planning as well as public health initiatives.

\section{Acknowledgments}

We thank all the participants in the CATSS for their contribution. S.S. research time was financed by grants from the Swedish state under the agreement between the Swedish government and the country councils, the ALF-agreement (ALFGBG-588611).

\section{Statement of Ethics}

All participants gave informed consent, and the study has been reviewed by an ethical review board (Dnr: 2010/1410-31/1).

\section{Disclosure Statement}

The authors have no conflicts of interest to declare.

\section{Funding Sources}

This study has not received direct funding.

\section{Author Contributions}

All authors fulfill the ICMJE criteria for authorship. S.S. has been responsible for conception and design of the work including analysis and interpretation as well as drafting the work, approving the final version, and being accountable for the work. H.K.C. has performed most of the statistical analysis and fulfills other criteria as well. E.L., S.L., and T.N. have all made substantial contributions to the interpretation of the data for the work, revised the work, approved of the final version, and agree on the accuracy and integrity of the work.

\section{References}

1 Whiteford HA, Degenhardt L, Rehm J, Baxter AJ, Ferrari AJ, Erskine HE, et al. Global burden of disease attributable to mental and substance use disorders: findings from the Global Burden of Disease Study 2010. Lancet. 2013 Nov;382(9904):1575-86.

2 Derringer J, Krueger RF, McGue M, Iacono WG. Genetic and environmental contributions to the diversity of substances used in adolescent twins: a longitudinal study of age and sex effects. Addiction. 2008 Oct;103(10):1744-51.

3 Palmer RH, McGeary JE, Francazio S, Raphael BJ, Lander AD, Heath AC, et al. The genetics of alcohol dependence: advancing towards systems-based approaches. Drug Alcohol Depend. 2012 Oct;125(3):179-91.

4 Langås AM, Malt UF, Opjordsmoen S. Indepth study of personality disorders in firstadmission patients with substance use disorders. BMC Psychiatry. 2012 Oct;12(1):180.

5 Garcia D, Lundström S, Brändström S, Råstam M, Cloninger CR, Kerekes N, et al. Temperament and character in the Child and Adolescent Twin Study in Sweden (CATSS): comparison to the general population, and genetic structure analysis. PLoS One. 2013 Aug;8(8):e70475.

6 Cloninger CR, Przybeck TR, Svrakic DM, Wetzel RD. The Temperament And Character Inventory (TCI): A Guide To Its Development And Use. St. Louis: Center for Psychobiology of Personality; 1994.

7 Svrakic DM, Whitehead C, Przybeck TR, Cloninger CR. Differential diagnosis of per- sonality disorders by the seven-factor model of temperament and character. Arch Gen Psychiatry. 1993 Dec;50(12):991-9.

8 Schneider R Jr, Ottoni GL, de Carvalho HW, Elisabetsky E, Lara DR. Temperament and character traits associated with the use of alcohol, cannabis, cocaine, benzodiazepines, and hallucinogens: evidence from a large Brazilian web survey. Br J Psychiatry. 2015 JanMar;37(1):31-9.

9 Arnau MM, Mondon S, Santacreu JJ. Using the temperament and character inventory (TCI) to predict outcome after inpatient detoxification during 100 days of outpatient treatment. Alcohol Alcohol. 2008 Sep-Oct; 43(5):583-8.

10 Campanella F, Crescentini C, Urgesi C, Fabbro F. Mindfulness-oriented meditation improves self-related character scales in healthy individuals. Compr Psychiatry. 2014 Jul; 55(5):1269-78.

11 Terock J, Janowitz D, Spitzer C, Miertsch M, Freyberger HJ, Grabe HJ. Alexithymia and self-directedness as predictors of psychopathology and psychotherapeutic treatment outcome. Compr Psychiatry. 2015 Oct;62:3441.

12 Anckarsäter H, Lundström S, Kollberg L, Kerekes N, Palm C, Carlström E, et al. The Child and Adolescent Twin Study in Sweden (CATSS). Twin Res Hum Genet. 2011 Dec; 14(6):495-508.

13 Quinn PD, Pettersson E, Lundström S, Anckarsäter $\mathrm{H}$, Långström N, Gumpert $\mathrm{CH}$, et al.
Childhood attention-deficit/hyperactivity disorder symptoms and the development of adolescent alcohol problems: A prospective, population-based study of Swedish twins. Am J Med Genet B Neuropsychiatr Genet. 2016 Oct;171(7):958-70.

14 Donahue K, Långström N, Lundström S, Lichtenstein P, Forsman M. Familial Factors, Victimization, and Psychological Health Among Sexual Minority Adolescents in Sweden. Am J Public Health. 2017 Feb;107(2): 322-8.

15 Babor T, Higgins-Biddle JC, Saunders JB, Monteiro MG. AUDIT - The alcohol use disorders identification test: guidelines for use in primary care. 2nd ed. Geneve: World Health Organization; 2001.

16 Hildebrand M. The Psychometric Properties of the Drug Use Disorders Identification Test (DUDIT): A Review of Recent Research. J Subst Abuse Treat. 2015 Jun;53:52-9.

17 Gasparrini A, Armstrong B, Kenward MG Distributed lag non-linear models. Stat Med. 2010 Sep;29(21):2224-34.

18 R core team. R: A Language and Environment for Statistical Computing [Internet]. Vienna, Austria: The $\mathrm{R}$ Foundation for Statistical Computing; 2017.

19 Le Bon O, Basiaux P, Streel E, Tecco J, Hanak C, Hansenne M, et al. Personality profile and drug of choice; a multivariate analysis using Cloninger's TCI on heroin addicts, alcoholics, and a random population group. Drug Alcohol Depend. 2004 Feb;73(2):175-82. 
20 Foulds JA, Mulder RT, Newton-Howes G, Adamson SJ, Boden JM, Sellman JD. Personality Predictors of Drinking Outcomes in Depressed Alcohol-Dependent Patients. Alcohol Alcohol. 2016 May;51(3):296-301.

21 Garcia D, Anckarsäter H, Lundström S. Self-directedness and cooperativeness, psychosocial dysfunction and suffering in ESSENCE. ScientificWorldJournal. 2013 Apr; 2013:416981.

22 Quednow BB, Hulka LM, Preller KH, Baumgartner MR, Eisenegger C, Vonmoos $\mathrm{M}$. Stable self-serving personality traits in recreational and dependent cocaine users. PLoS One. 2017 Mar; 12(3):e0172853.

23 Grant BF, Chou SP, Saha TD, Pickering RP, Kerridge BT, Ruan WJ, et al. Prevalence of 12-Month Alcohol Use, High-Risk Drinking, and DSM-IV Alcohol Use Disorder in the United States, 2001-2002 to 2012-2013: Results From the National Epidemiologic Survey on Alcohol and Related Conditions. JAMA Psychiatry. 2017 Sep;74(9):911-23.

24 Steingrímsson S, Carlsen HK, Sigfússon S, Magnússon A. The changing gender gap in substance use disorder: a total populationbased study of psychiatric in-patients. Addiction. 2012 Nov;107(11):1957-62.

25 Carliner H, Mauro PM, Brown QL, Shmulewitz D, Rahim-Juwel R, Sarvet AL, et al. The widening gender gap in marijuana use prevalence in the U.S. during a period of economic change, 2002-2014. Drug Alcohol Depend. 2017 Jan; 170:51-8.

26 Montag C, Jurkiewicz M, Reuter M. Low self-directedness is a better predictor for problematic internet use than high neuroticism. Comput Human Behav. 2010;26(6): 1531-5.

27 Del Pino-Gutiérrez A, Fernández-Aranda F, Granero R, Tárrega S, Valdepérez A, Agüera $\mathrm{Z}$, et al. Impact of alcohol consumption on clinical aspects of gambling disorder. Int J Ment Health Nurs. 2017 Apr;26(2):121-8.

28 Zaaijer ER, Bruijel J, Blanken P, Hendriks V, Koeter MW, Kreek MJ, et al. Personality as a risk factor for illicit opioid use and a protective factor for illicit opioid dependence. Drug Alcohol Depend. 2014 Dec;145:101-5.

29 Liskola J, Haravuori H, Lindberg N, Niemelä S, Karlsson L, Kiviruusu O, et al. AUDIT and AUDIT-C as screening instruments for alcohol problem use in adolescents. Drug Alcohol Depend. 2018 Jul;188:266-73. 